\title{
There goes the neighbourhood
}

The realization that the tumour microenvironment plays more than just a supporting role in tumour progression represented a radical shift in the study of tumour biology. Although the profound influence of the stroma on tumorigenesis is now widely accepted, a full understanding of the interactions between a tumour and its surroundings remains a nascent field. Yang and colleagues provide further evidence that signals emanating from the microenvironment, in this case infiltrating mast cells, impact neurofibroma progression.

Neurofibromas are composed of a plethora of cell types, including Schwann cells, endothelial cells, fibroblasts and mast cells. Biallelic loss of neurofibromin 1 (NF1), the gene that encodes the GTPase activating protein neurofibromin, in the embryonic Schwann cell lineage is necessary for neurofibroma development, but recent data have also pointed to a crucial role for NF1 in the tumour stroma. In support of this notion, a team led by Luis Parada and D. Wade Clapp showed that introduction of bone marrow from $\mathrm{Nf}^{+/-}$but not wild-type mice into recipients that harboured $N f 1$ deficient Schwann cells resulted in neurofibroma formation, indicating that $N f 1$ heterozygosity in the bone marrow is a second prerequisite for the genesis of these tumours.

Which component of the bone marrow is responsible? Mast cell infiltration is an established feature of neurofibromas, and the authors had shown in previous in vitro studies that Nf1-heterozygous mast cells were recruited by and could engage in crosstalk with $\mathrm{Nf1}$-deficient Schwann cells. Consistent with these findings, the mast cells that were present in the in vivo neurofibromas were all $\mathrm{Nf1}^{+-}$rather than wild type. To ensure that this recruitment was actively required for, rather than a

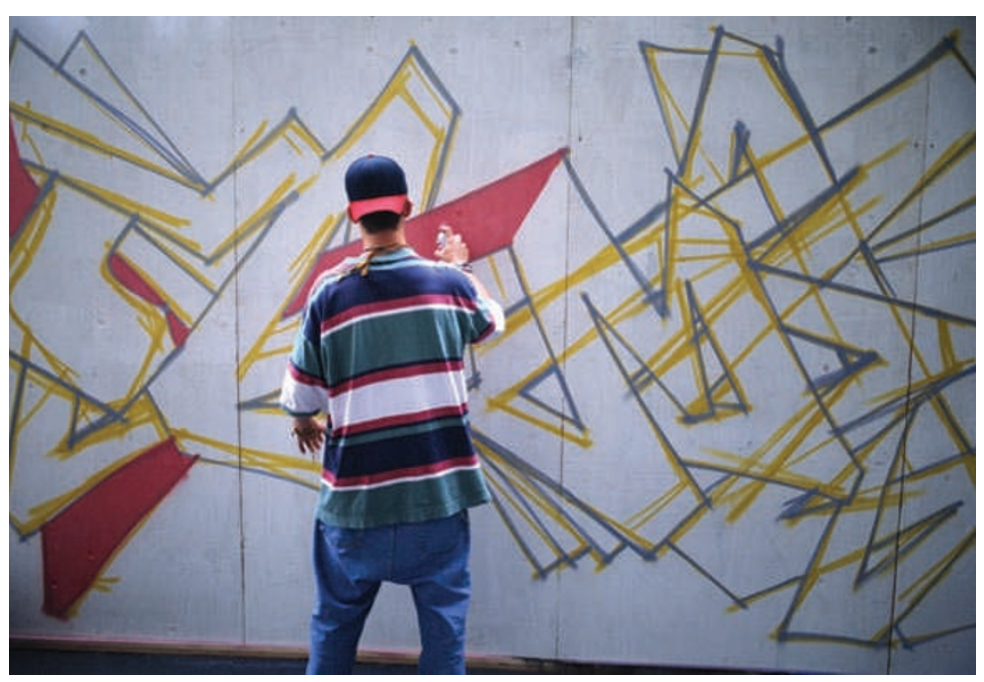

\section{...targeting the tumour microenviroment can be as effective as targeting the tumour itself.}

consequence of, tumorigenesis, the authors exploited the dependence of mast cells on KIT signalling for their development and survival. In mice, the small-molecule KIT inhibitor imatinib mesylate led to a decrease in mast cell occupation of neurofibromas together with significant tumour regression but, more importantly, it also resulted in profound tumour shrinkage in a 3-year-old patient with life-threatening neurofibroma.

The authors concede that more extensive clinical trials need to be conducted before imatinib mesylate could be accepted as a bona fide therapeutic option for patients with neurofibroma, but their data certainly suggest that KIT inhibition might be a novel target in the treatment of this disease. Questions raised by the study, such as how mast cells contribute to tumour development, will also need to be addressed. For now, however, these results are a powerful illustration that targeting the tumour microenvironment can be as effective as targeting the tumour itself.

Safia Ali Danovi

ORIGINAL RESEARCH PAPER Yang, F. et al. Nf1-dependent tumours require a microenvironment containing $\mathrm{Nf}^{+/ /}$- and c-kitdependent bone marrow. Cell 135, 437-448 (2008) 